\title{
The Role of MiR-5094 as a Proliferation Suppressor during Cellular Radiation Response via Downregulating STAT5b
}

Nan Ding, ${ }^{1}$ Junrui Hua ${ }^{1}$, Jinpeng He ${ }^{1}$, Dong Lu' ${ }^{1}$, Wenjun Wei ${ }^{1}$, Yanan Zhang ${ }^{1}$, Heng Zhou ${ }^{1}$, Liying Zhang ${ }^{2}$, Yongqi Liu², Guangming Zhou ${ }^{3}$, Jufang Wang ${ }^{\circledR}$

1. Key Laboratory of Space Radiobiology of Gansu Province \& Key Laboratory of Heavy Ion Radiation Biology and Medicine of Chinese Academy of Sciences, Institute of Modern Physics, Chinese Academy of Sciences, Lanzhou 730000, China

2. Gansu University of Traditional Chinese Medicine, Lanzhou 730000, China

3. Medical College of Soochow University, Suzhou 215123, China

$\bowtie$ Corresponding author: Jufang Wang, Institute of Modern Physics, Chinese Academy of Science, 509 Nanchang Road, Lanzhou 730000, China. Email: jufangwang@impcas.ac.cn

(c) The author(s). This is an open access article distributed under the terms of the Creative Commons Attribution License (https://creativecommons.org/licenses/by/4.0/). See http://ivyspring.com/terms for full terms and conditions.

Received: 2019.08.27; Accepted: 2019.12.22; Published: 2020.02.03

\begin{abstract}
MicroRNAs (miRNAs) play important roles in the regulation of cellular stress responses. We previously uncovered 10 novel human miRNAs which are induced by X-ray irradiation in HeLa cells using Solexa deep sequencing. The most highly expressed new miRNA, miR-5094, was predicted to target STAT5b. This study wonders whether miR-5094 participates in cellular radiation response via STAT5b. Firstly, direct interaction between miRNA-5094 and the STAT5b 3'-UTR was confirmed by luciferase reporter assay. Then, the radiation responsive expression of miR-5094 and STAT5b were measured in HeLa and Jurkat cells, and the expressions of down-stream genes of STAT5b after ionizing radiation (IR) were detected in HeLa cells. At last, the effects of miR-5094 on survival fraction, cell proliferation, cell cycle arrest and apoptosis induced by IR were investigated in HeLa cells, Jurkat cells and human peripheral blood T cells. It was found that up-regulation of miR-5094 by radiation induction or miRNA mimic transfection suppressed expression of STAT5b, and consequently decreased the transcription of down-stream Igf-1 and Bcl-2. Additionally, over expression of miR-5094 resulted in proliferation suppression and knockdown of miR-5094 by miRNA inhibitor after irradiation partially reversed the proliferation suppression induced by miR-5094 in HeLa cells, Jurkat cells and $\mathrm{CD}^{+} \mathrm{T}$ cells. Collectively, our findings demonstrate that up-regulation of miR-5094 down-regulated the expression of STAT5b, thereby suppressing cell proliferation after X-ray irradiation.
\end{abstract}

Key words: microRNA, radiation, miR-5094, STAT5b, proliferation

\section{Introduction}

Radiation therapy (RT) is one of the three primary modalities of cancer treatment. RT uses ionizing radiations (IR) to cure tumor patients by IR-induced direct and indirect DNA damages, on the basis of classic target theory. Mammalian cells respond to IR-induced DNA damages by activating sophisticated DNA damage response (DDR), which includes cell cycle arrest, activation of DNA repair genes, and triggering of apoptosis [1].

RT also modulates the tumor microenvironment [2]. In recent studies, RT has been demonstrated to induce the activation of $\mathrm{T}$ cells, which in turn cause immunogenic cell death of cancer cells [3]. Growing number of researches described the combination of radiotherapy with immunotherapy. For instance, immune checkpoint inhibitors (ICI), make it possible to enhance antitumor effects $[4,5]$. It is important to consider the biology of radiation response of immune system in developing strategies of combined radiotherapy with immunotherapy.

Up-regulation and down-regulation of microRNAs (miRNAs) represent unique cellular radiation responses. MiRNAs are a class of endogenous, short ( $\sim 22$ nucleotides) and single-stranded non-coding 
RNAs, which act as negative regulators of gene expression at post-transcriptional level. MiRNAs bind to the $3^{\prime}$ untranslated region (3'UTR) of mRNA of their targets, and subsequently promoting mRNA degradation and/or inhibiting translation [6]. As powerful post-transcriptional regulators, miRNAs are broadly involved in regulation of radiation-related damage repair processes [7-9]. In last two decades, a larger number of radiation responsive miRNAs were detected by miRNA microarray and deep sequencing technology [10-13]. A lot of radiation responsive miRNAs were confirmed to regulate cellular radiation sensitivity by manipulating expression of DNA damage repair regulators [11-13]. For example, a p53-dependent up-regulation of miR-34 after irradiation was reported both in vivo and in vitro, and, the miR-34 down-regulated Bcl-2 and Notch, which in turn sensitized cancer cells to radiation [14, 15]. In our previous study, 190 up-regulated miRNAs and 231 down-regulated miRNAs were detected in HeLa cells after 2 Gy X-ray irradiation using Solexa sequencing, and 10 novel human miRNAs were identified by qRT-PCR [16]. Among these 10 miRNAs, miR-5094 was the most abundantly expressed one after radiation treatment, which was predicted to target signal transducers and activators of transcription $5 b$ (STAT5b) [16].

STAT5b is an important member of the Janus kinase/signal transducers and activators of transcription (JAK/STAT) signaling pathway which plays key biological roles in growth, immune responses and cancers $[17,18]$. As a universal transcription factor, STAT5b is stimulated by various cytokines including growth hormones (GH) and interleukins [19]. Particularly, STAT5b is a key mediator of GHregulated Igf-I transcription which in turn influence cell growth both in vivo and in vitro [20]. STAT5b was also reported to regulate cell growth, cell cycle progression and apoptosis in human glioblastoma and breast cancer cells through its down-stream genes like Bcl-2, p21 and Cyclin D1 [21-23]. Furthermore, $\mathrm{CD}^{+}$and $\mathrm{CD}^{+}{ }^{+} \mathrm{T}$ cells from the STAT5b transgenic NOD mice showed a higher proliferation capacity, and the number and proportion of $\mathrm{CD} 4{ }^{+} \mathrm{CD} 25^{+}$ regulatory $\mathrm{T}$ cells were significantly increased [24]. Conversely, in STAT5b-deficient patient, deficiency of STAT5b function displayed immune dysregulation and decreased numbers of $\mathrm{CD} 4{ }^{+} \mathrm{CD} 25^{\text {high }} \mathrm{T}$ cells [25].

Several studies reported that the expression and activation of STAT5b were modulated by IR and UV radiation (UVR). It was demonstrated that UVR inhibited IL-2-induced activation of STAT5 in T lymphocytes [26]. Likewise, down-regulation of STAT5b expression was detected in ATM mutated human fibroblast AT5BIVA cells at half hour after 10
Gy $\gamma$-ray irradiation [27]. However, up-regulation of STAT5b was detected in peripheral blood of neuroblastoma patients $72 \mathrm{~h}$ after Medical Internal Radiation Dose (MIRD) schema [28]. Also, the expression of STAT5b was slightly increased in both directly irradiated and bystander k562 cells at one hour after 4 Gy of X-ray irradiation [29]. Yet, the underlying mechanism of STAT5b aberrant expression during cellular radiation response is unknown.

Several miRNAs have been described to target STAT5b. It was reported that miR-200a directly repressed STAT5b expression in both mice and human [30], while miR-134 was showed to inhibit proliferation, survival and xenograft growth in cancer cell and stem-cell by targeting STAT5b and KRAS [31]. Similarly, miR-150 was demonstrated to suppress expression of STAT5b in mammary epithelial cells of bitransgenic mice [32]. Whether and how miRNAs regulate STAT5b in DNA damage response to IR remains to be an open question.

In present study, we validated STAT5b as a target of miR-5094. We demonstrated an inhibition of STAT5b expression by radiation-induced miR-5094 and uncovered suppressing effect of miR-5094 on proliferation of different cells, especially on proliferation of $\mathrm{CD}^{+} \mathrm{T}$ cells and $\mathrm{CD} 4{ }^{+} \mathrm{CD} 25^{+}$Treg cells during cellular radiation response. Our study for first time provided evidences that radiation- induced miR-5094 participates in cellular radiation response via targeting STAT5b.

\section{Materials and Methods}

\section{Cell lines}

Human cervical carcinoma cell line HeLa, Epstein-Barr virus transformed B cells (EBV-B) and human acute $\mathrm{T}$ cell leukemia Jurkat cells were cultured in RPMI-1640 medium (Gibco, 31800-105, USA), while human bronchial epithelial cell Beas-2B was cultured in DMEM medium (Gibco, 12800-082). Both RPMI-1640 and DMEM medium were supplemented with 10\% fetal bovine serum (Gibco, 10099-141), 100 units/mL penicillin and $100 \mathrm{mg} / \mathrm{mL}$ streptomycin, and, the cells were maintained at $37^{\circ} \mathrm{C}$ in a humidified atmosphere of $95 \%$ air and $5 \% \mathrm{CO}_{2}$.

\section{The generation of $\mathrm{CD4}^{+} \mathrm{T}$ cells and CD4+CD25+ Treg cells}

The study protocol was approved by the medical ethics committee of affiliation hospital of Gansu University of Chinese Medicine and the informed written consents were obtained from all volunteers. Peripheral blood was obtained from healthy volunteers and the peripheral blood mononuclear cells (PBMCs) were isolated by density centrifugation 
over Ficoll (Histopaque 1077, Sigma Aldrich, USA). $\mathrm{CD}^{+} \mathrm{T}$ cells were enriched with an isolation kit $\left(\mathrm{CD} 4^{+}\right.$ $\mathrm{T}$ Cell Isolation Kit human, Miltenyi Biotec, Auburn, CA, USA), which contains a cocktail of CD8a, CD14, CD15, CD16, CD19, CD36, CD56, CD123, TcR $/ \delta$, and CD235a (Glycophorin A) antibodies. The cell separation was performed with LS columns (Miltenyi Biotech), according to the manufacturer's instructions. The $\mathrm{CD}^{+}$cells were further labeled with PEconjugated anti-CD25 mAb (BD Bioscience, CA 555432, USA), PerCP-conjugated anti-CD4 mAb (BD Bioscience, 566321) and FITC-conjugated anti-CD127 $\mathrm{mAb}$ (BD Bioscience, 560549). The $\mathrm{CD}^{+} \mathrm{CD} 25^{+}$Treg cells were obtained by cell sorting (purity $>95 \%$ ) on MoFlo XDP (Beckman coulter, Pasadena, CA, USA). $\mathrm{CD} 4{ }^{+}$cells and $\mathrm{CD} 4{ }^{+} \mathrm{CD} 25^{+}$cells were stimulated with anti-CD3/CD28-coated beads (Gibco, 11161D) (1:4), IL-2 (Gibco, PHC0026) (20 U/mL) in AIM-V (Gibco, 12055083) serum-free medium in 12 -well plates (BD Labware).

\section{Cell transfection and irradiation}

For HeLa and Beas-2B cells, $2 \times 10^{5}$ cells were seeded in $35 \mathrm{~mm}$ dishes and cultured for $24 \mathrm{~h}$ prior to be transfected with small RNAs including miR-5094 mimics (GeneCopoeia, HmiR1458, China), negative control (GeneCopoeia, CmiR0001-MR04), miR-5094 inhibitor (GeneCopoeia, HmiR-AN2605), and the inhibitor negative control (GeneCopoeia, CmiRAN0001-AM01) or STAT5b siRNA (RioNeer, 1145682, 1145685, 1145687, CA, USA) and its negative control (BioNeer, Daejeon, Korea) at 40-60\% confluence using Lipofectamine TM 2000 (Invitrogen, 11668-019, USA). Five hours after transfection, the cells were irradiated with a laboratory RX-650 X-ray source (Faxitron, Tucson, Arizona, USA), at a dose rate of $0.8 \mathrm{~Gy} / \mathrm{min}$ (100 keV, 5mA). For EBV-B cells, Jurkat cells, CD4 ${ }^{+} \mathrm{T}$ cells and $\mathrm{CD} 4{ }^{+} \mathrm{CD} 25^{+}$Treg cells, $5 \times 10^{5}$ cells in $0.5 \mathrm{~mL}$ OPTI-MEM were transfected with different RNAs using Entranster ${ }^{\mathrm{TM}}-\mathrm{R} 4000$ transfection reagent [33] (EnGreen Biosystem, 4000-4, Beijing, China) in $1.5 \mathrm{~mL}$ Eppendorf tube. The transfection was done for $5 \mathrm{~h}$ with the final concentration of the miRNA mimics or inhibitor at $30 \mathrm{nM}$, and the siRNAs concentration were at $50 \mathrm{nM}$. The transfected cells were irradiated at a dose rate of $0.8 \mathrm{~Gy} / \mathrm{min}(100 \mathrm{keV}, 5 \mathrm{~mA})$. At last, cells were centrifuged and reseeded in 12-well plates with fresh culture medium.

\section{Luciferase reporter assay}

STAT5b 3'-UTRs containing wild type or mutant miR-5094 binding sites, were synthesized by Sangon Biotechnology Co. (Shanghai, China). The segments were inserted into pmirGLO DualLuciferase miRNA Target Expression Vectors
(Promega, WI, USA). For luciferase reporter assay, $1.5 \times 10^{5} \mathrm{HeLa}$ cells were seeded and grown in 12-well plate for $24 \mathrm{~h}$, and then the cells were co-transfected with 300 ng DNA (pmirGLO-3' UTR constructs or derived mutants) and $30 \mathrm{nM}$ of miR-5094 mimics (GeneCopoeia, HmiR1458) or negative control (GeneCopoeia, CmiR0001-MR04) using Lipofectamine TM 2000 (Invitrogen, 11668-019). Dual luciferase assays were performed $24 \mathrm{~h}$ after transfection using Dual Luciferase Reporter Assay Kit (Promega, E1910) with Tecan Infinite M200 Pro microplate reader (Tecan, Mannedorf, Switzerland).

\section{qRT-PCR}

Total RNA was extracted using TRIzol reagent (Invitrogen, 15596018). Reverse transcription of miRNA or mRNA was conducted with All-in-OneTM miRNA First-Strand cDNA Synthesis kit (GeneCopoeia, QP014) or All-in-OneTM First-Strand cDNA Synthesis kit (GeneCopoeia, AORT-0060). To quantify the miR-5094 expression or mRNA expression of STAT5b, Bcl-2, Cyclin D1, Igf-1 and p21, real-time PCR was performed on CFX96 Touch ${ }^{\mathrm{TM}}$ Real-Time PCR Detection System (Bio-Rad, California, USA) using All-inOneTM miRNA qPCR kit (GeneCopoeia, AMPR-0200) or Allin-OneTM mRNA Detection kit (GeneCopoeia, AOPR-0200) based on SYBR-Green. PCR primers for U6 (HmiRQP9001), miR-5094 (HmiRQP2605), miR-34a (HmiRQP0439), miR-134 (HmiRQP3060), miR-150-5p (HmiRQP0209), miR200a (HmiRQP0297), GAPDH (HQP006940), STAT5b (HQP017774), Bcl-2 (HQP016211), Cyclin D1 (HQP01 6204), Igf-1 (HQP009518) and p21 (HQP000331) were all purchased from GeneCopoeia. In each qRT-PCR, snRNA U6 and GAPDH mRNAs were used as control for normalization respectively. The relative expression was calculated using the $2^{-\Delta \Delta \mathrm{Ct}}$ method.

\section{Western blotting analysis}

Protein lysates were made in RIPA buffer (Beyotime, P0013C, China). Proteins were separated by $12 \%$ SDS-PAGE electrophoresis and transferred to a methanol-activated PVDF membrane (Millipore, IPVH00010, USA). The membrane was blocked with $5 \%$ milk in PBS containing 0.1\% Tween-20 for $1 \mathrm{~h}$ and subsequently probed with anti-STAT5b antibody (Abcam, ab226193, MA, USA), anti-pSTAT5b antibody (Abcam, ab52211), anti-beta Actin antibody (Abcam, ab228001), anti-Bcl2 antibody (Abgent, AP20322c, China), anti-Cyclin D1 antibody (Abcam, ab134175), anti-Igf1 antibody (Abgent, AP14099b) or p21 antibody (Santa Cruz, sc-397) at room temperature for $2 \mathrm{~h}$. After $1 \mathrm{~h}$ incubation with goatanti-rabbit HRP-conjugated secondary antibody (Abcam, ab97051) or goat-anti-mouse HRP- 
conjugated secondary antibody (Abcam, ab97023), the protein bands were detected with luminal reagent (Millipore, WBKLS0500, USA).

\section{Colony formation assay}

HeLa cells were irradiated with 2 Gy of X-rays right after RNA-transfection. Then, cells were harvested by trypsinization and then re-suspended in RPMI-1640 medium supplemented with 10\% FBS. An appropriate number of cells were plated into a $60 \mathrm{~mm}$ dish to produce $\sim 50-150$ colonies. After grown for 10 days, the cells were fixed with $75 \%$ ethanol and stained with $0.5 \%$ crystal violet at room temperature. The cell colonies containing more than 50 cells were counted and at least three parallel dishes were scored for each treatment. The experiments were conducted independently for more than three times.

\section{Cell Viability assay}

Cell viability was determined by MTT assay. Briefly, HeLa cells were seeded into 96-well plates a day before RNA transfection. The RNA-transfected cells were exposed to 2 Gy of X-rays and allowed to grow for $48 \mathrm{~h}$. Then, $10 \mu \mathrm{L}$ of MTT solution at $0.5 \mathrm{mg} /$ $\mathrm{mL}$ was added in wells and incubated for $4 \mathrm{~h}$ at $37^{\circ} \mathrm{C}$. The medium was replaced with $100 \mu \mathrm{L}$ dimethylsulfoxide and vortexed for 10 minutes. The optical density (OD) values at $570 \mathrm{~nm}$ were determined by Tecan Infinite M200 Pro microplate reader.

\section{Cell growth curves}

$2 \times 10^{5}$ Jurkat cells, $\mathrm{CD} 4^{+} \mathrm{T}$ cells or $\mathrm{CD} 4^{+} \mathrm{CD} 25^{+}$ Treg cells were exposed to 2 Gy of X-rays after RNA transfection, and then centrifuged and reseeded in 12-well plates with fresh medium. $\mathrm{CD}^{+} \mathrm{T}$ cells or $\mathrm{CD} 4{ }^{+} \mathrm{CD} 25^{+}$Treg cells were stimulated with antiCD3/CD28 and IL-2 at same time. The cell growth curves were plotted with the cell numbers counted by Coulter Counter (Beckman, Brea, CA, USA).

\section{Flow cytometry}

For cell cycle assay, cells were first exposed to IR at a proper dose. Twenty-four hours post-irradiation, the cells were then fixed in $-20^{\circ} \mathrm{C}$ prechilled $70 \%$ alcohol overnight, and stained with $20 \mu \mathrm{g} / \mathrm{mL}$ propidium iodide (PI) at room temperature. For apoptosis assay, cells were harvested $24 \mathrm{~h}$ post-irradiation and stained with Annexin V-FITC/PI (BD Biosciences, 556547) for $30 \mathrm{~min}$ at room temperature. For $\mathrm{CD}^{+}{ }^{+} \mathrm{CD} 25^{+}$Treg percentage assay, cells were labeled with PE-conjugated anti-CD25 $\mathrm{mAb}$ and FITCconjugated anti-CD4 mAb (BD Bioscience, 550628).

Flow cytometry assay was performed using an Amnis imaging flow cytometer (Merck Millipore, Darmstadt, Hesse, Germany) and at least 10,000 gated events were acquired from each sample. The data were analyzed with IDEAS Application v6.0 (Amnis) or FlowJo v6.0 (Tree Star, Ashland, OR, USA).

\section{Statistical analysis}

All the experiments were repeated at least three times and results are shown as mean \pm SD. The statistical significance of the results was determined by Student's t-test and $\mathrm{P}<0.05$ was considered as a statistically significant difference.

\section{Results}

\section{MiR-5094 directly targets STAT5b}

We previously demonstrated the up-regulation of miR-5094 induced by ionizing radiation and predicted the STAT5b as a targets of miR-5094 by TargetScan software [16]. In this study, to determine whether miR-5094 directly targets STAT5b via such a 3'-UTR, we synthesized two kinds of STAT5b 3'-UTR fragments which contain either wild-type or mutated putative miR-5094 seed region (Figure 1A), then cloned them into pmirGLO luciferase report vectors and conducted the reporter gene assay respectively. Co-transfection of luciferase reporter containing wild type STAT5b 3'-UTR and miR-5094 mimics into HeLa cells significantly repressed the luciferase activity by approximately $60 \%(P=0.015)$, while suppression of luciferase activity was abolished when a mismatch mutation was introduced in the putative binding sites of STAT5b 3'-UTR (Figure 1B).

Next, we validated the inhibition of STAT5b expression by miR-5094. As shown in Figure 1C and 1D, the miR-5094 mimics specifically suppressed both the STAT5b protein and mRNA expressions at $24 \mathrm{~h}$ post-transfection in HeLa cells, Beas-2B cells, EBV-B cells and Jurkat cells. Transient transfection of HeLa cells with miR-5094 inhibitor suppressed expression of miR-5094 and resulted in an increasing of STAT5b mRNA (Figure 1C). As expected, HeLa cells transfected with STAT5b siRNA showed remarkably decrease in STAT5b expression in both transcriptional levels (Figure 1C) and translational levels (Figure 1D).

\section{lonizing radiation-induced $\mathrm{miR}-5094$ expression results in STAT5b suppression}

To investigate the expression profiles of miR-5094 and STAT5b under ionizing irradiation, the kinetics of miR-5094 or STAT5b expression was monitored by quantitative RT-PCR and Western blotting in 2 Gy X-ray irradiated HeLa cells. Expression of miR-5094 increased immediately after radiation and peaked at about $4 \mathrm{~h}$ after IR treatment, then declined until $48 \mathrm{~h}$. Levels of STAT5b mRNA and protein decreased gradually after irradiation and the lowest point was detected at about $4 \mathrm{~h}$ (Figure 2A). We further examined miR-5094 and STAT5b mRNA 
expression under different radiation dosages. As shown in Figure 2B, a clear increase in miR-5094 and decrease of STAT5b were detected under all tested doses. At $4 \mathrm{~h}$, the expression of miR-5094 increased with the rising of radiation dose, and peaked at about 8 Gy. Nevertheless, the decrease of STAT5b did not show a clear dose response.

We also checked expression of miR-134, miR-150-5p and miR-200a, which reportedly regulate STAT5b expression [30-32] in X-ray irradiation HeLa cells. The expression of miR-34a, who is up-regulated after irradiation [14], was used as a reference of radiation treatment. As shown in Figures 2C, among miR-134, miR-150-5p and miR-200a, only miR-150-5p was slightly increased but without statistical significance. On the other hand, the expression of miR-34a was significantly increased at both $12 \mathrm{~h}$ and $24 \mathrm{~h}$ after irradiation.

\section{MiR-5094 changes the expression of STAT5b down-stream regulators}

To investigate whether miR-5094 up-regulation affects the expression of STAT5b down-stream genes, we examined expression of Bcl-2, Cyclin D1, Igf-1 and p21, which are known to be regulated by STAT5b and have important roles during cell radiation response [20-22]. As shown in Figure 3A, 2 Gy X-rays increased the expression of miR-5094 and decreased the transcription of STAT5b mRNA. Meanwhile, transcription of Bcl-2 and Igf-1 was clearly decreased after transient transfection of miR-5094 mimics or STAT5b siRNA and IR radiation. The Cyclin D1 mRNA was significantly affected by STAT5b siRNA but not by miR-5094 mimics or radiation. Moreover, the expression of p21 was remarkably up-regulated by X-ray irradiation, or by miR-5094 mimics or STAT5b siRNA transfection. More importantly, miR-5094 inhibitor rescued the decline of STAT5b after radiation treatment, and subsequently upregulated the expression of down-stream Bcl-2 and Igf-1, but not significantly influenced expression of Cyclin D1 and p21 (Figure 3A).

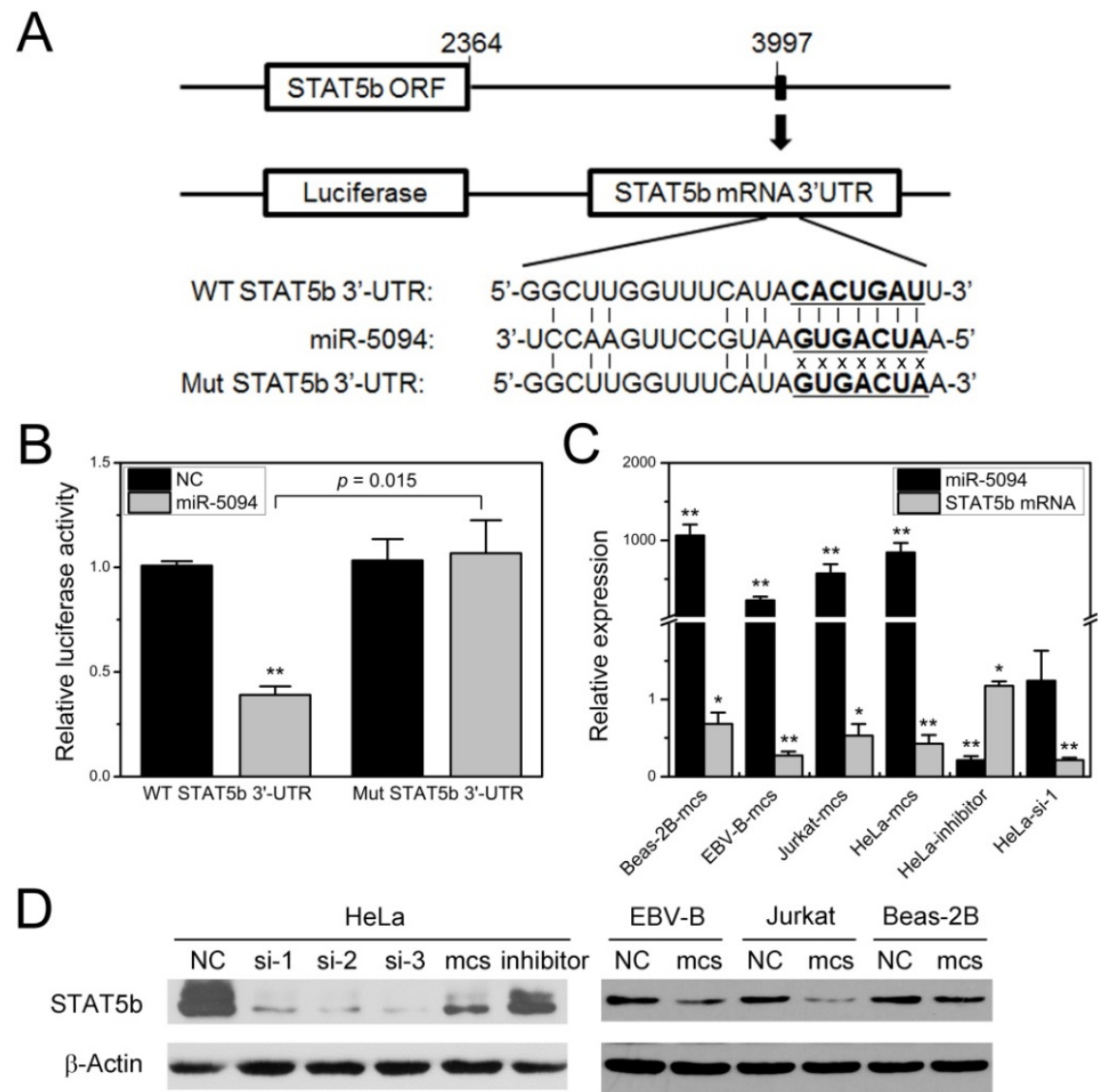

Figure 1. MiR-5094 directly targets STAT5b. (A) Alignment of wild-type seed sequence of the 3'-UTR of STAT5b mRNA (WT STAT5b 3'-UTR) and a mutated seed sequence of the miR-5094-binding site (Mut STAT5b 3'-UTR). The seed region is shown in bold. (B) Luciferase reporter assays. Luciferase reporter containing wild-type or mutant STAT5b 3'UTR was co-transfected with exogenous miR-5094 mimics (miR-5094) or negative mock control (NC) into HeLa cells. Luciferase activity was measured $24 \mathrm{~h}$ after transfection. Renilla luciferase activity was used to normalize the firefly luciferase activity. (C) MiR-5094 suppresses STAT5b mRNA expression in different cells at $24 \mathrm{~h}$ after transfection. The relative expression levels were normalized to same cells transient transfected with NC at same time point. (D) MiR-5094 suppresses STAT5b protein expression in different cells at $24 \mathrm{~h}$ after transfection. Mcs: miR-5094 mimics; inhibitor: miR-5094 inhibitor; si-1, si-2 and si-3: STAT5b siRNA. *P < 0.05 and **P < 0.01 represent the comparison with NC. 
A
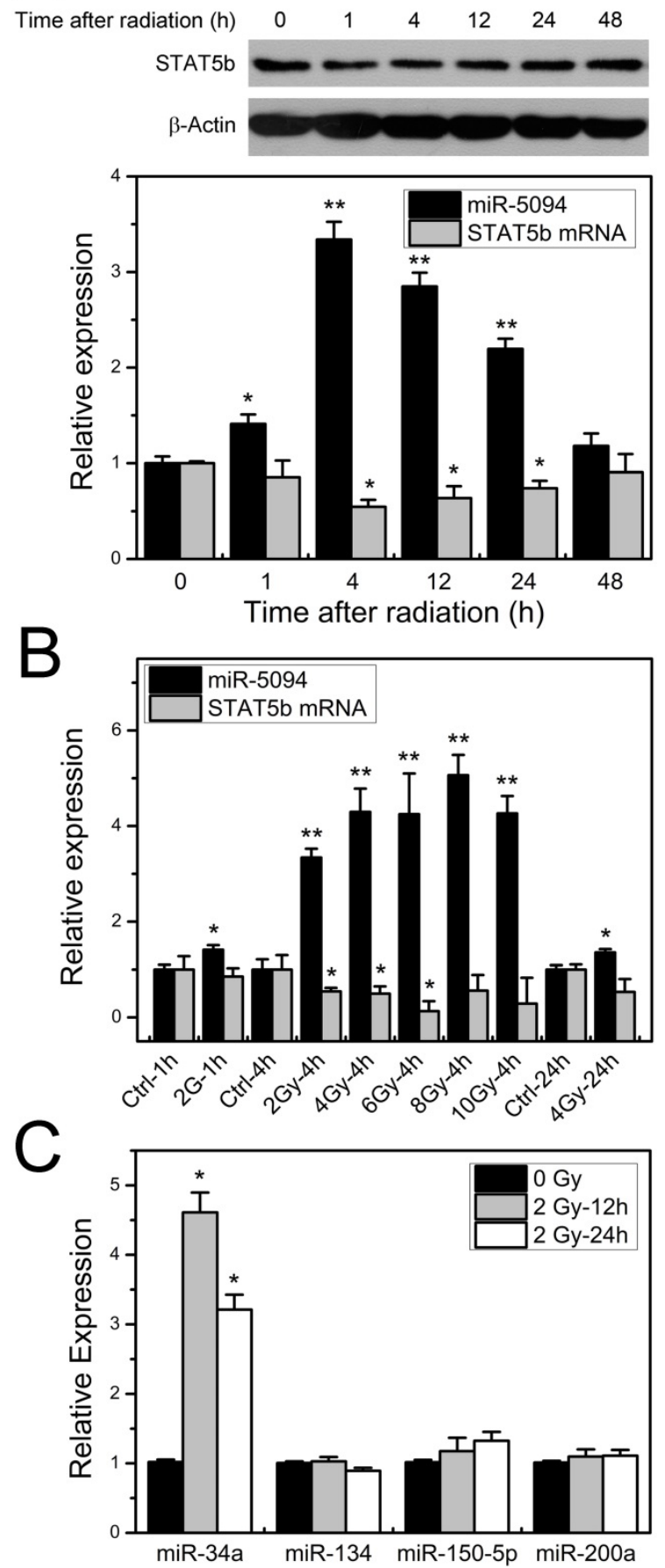

Figure 2. Radiation induces increase expression of miR-5094 and decrease expression of STAT5b. (A) STAT5b and miR-5094 expression in HeLa cells at different time points after radiation. U6 was used as control of miR-5094 expression, and GAPDH mRNA was used as control of STAT5b mRNA. (B) Expression of miR-5094 and STAT5b mRNA in HeLa cells after different dosages of irradiation treatment. U6 and GAPDH were used as controls. (C) Expression of miR-34a, miR-134, miR-150-5p and miR-200a after radiation in HeLa cells. The qRT-PCR was conducted to quantify the expression levels of miR-34a, miR-134, miR-150-5p and miR-200a at $12 \mathrm{~h}$ and $24 \mathrm{~h}$ after $2 \mathrm{~Gy} \mathrm{X}$-rays. U6 was used as controls. $* \mathrm{P}<0.05$ or **P $<0.01$ represents statistical significance of comparison against non-irradiated control.

Furthermore, translation levels of these genes were also detected. As shown in Figure 3B, radiation treatment suppressed the expression of STAT5b, and down-regulated expression of Bcl-2, Cyclin D1 and Igf- 1 . On the contrary, the expression of p21 was up-regulated by radiation. As expect, the transfection of miR-5094 mimics or STAT5b siRNA resulted in a down-regulation of STAT5b, Bcl-2, Cyclin D1 and Igf-1, and up-regulation of p21. Moreover, the transfection of miR-5094 inhibitor rescued the expression of STAT5b, Bcl-2, Igf-1 and Cyclin D1 after radiation compared with $\mathrm{NC}$.

\section{MiR-5094 dampen down proliferation after radiation}

To investigate the role of miR-5094 in regulation of the cellular response to IR, the intracellular miR-5094 levels were up/downregulated by transient transfection with miR-5094 mimics (mcs) or inhibitor. Cell survival, cell viability, cell cycle distribution, cell proliferation and apoptosis were monitored after IR.

As shown in Figure 4A, compared with non-irradiated groups, $\mathrm{X}$-ray irradiation significantly decreased HeLa cells survival fraction. Transfection of miR-5094 mimics or STAT5b siRNA remarkably exacerbated the decrease of cell survival after X-ray irradiation, while miRNA inhibitor showed no significant effect on cell survival. Meanwhile, the transfection of STAT5b siRNA induced a moderate decrease of cell survival even without X-ray irradiation.

The cell viability assay showed that radiation markedly suppressed cell viability of HeLa cells. Moreover, like STAT5b siRNA, miR-5094 mimics significantly decreased cell viability, and exaggerated the decrease of cell viability after radiation. Notably, the inhibition of miR-5094 by miRNA inhibitor significantly recovered the decrease of cell viability, as compared with NC transfection group after irradiation (Figure 4B).

The apoptotic assay showed that X-ray irradiation induced significant increase in apoptosis of HeLa cells. Transfection of STAT5b siRNA resulted in an increasing of apoptosis in un-irradiated HeLa cells and exacerbated the apoptosis of HeLa cells after X-ray irradiation (Figure 4C). As compared with negative control, miR-5094 mimics also significantly increased apoptosis of HeLa cells after X-ray irradiation, while less effective than STAT5b siRNA. The miR-5094 inhibitor transfection did not affect non-irradiated cells. However, the inhibitor decreased apoptosis rate of cells compared with negative control after radiation (Figure 4C).

The cell cycle assay showed that X-ray irradiation induced significant G2/M arrested (Figure 4D). However, the transfection of miR-5094 mimics, STAT5b siRNA or miR-5094 inhibitor showed no significant influence on cell cycle distributions with or without radiation treatment (figure 4D). 
A

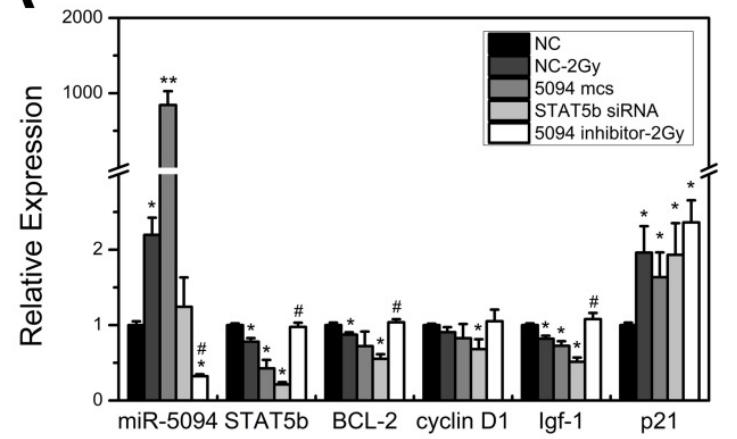

B
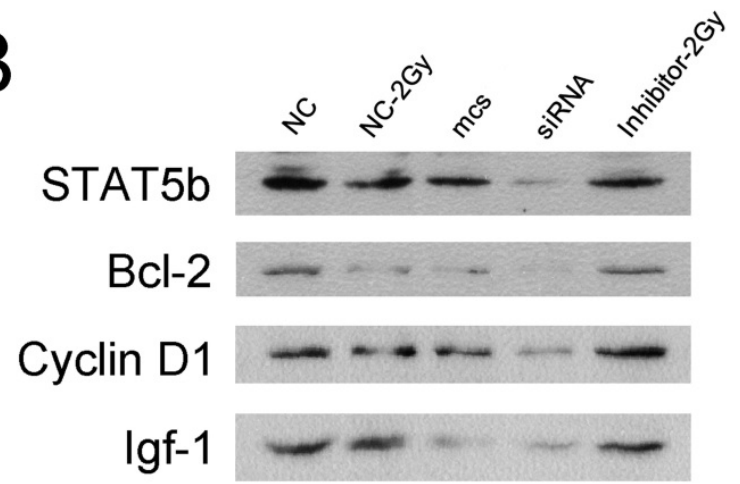

p21
$\beta$-Actin

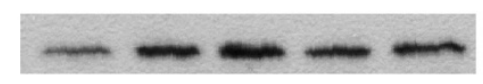

Figure 3. Influence of miR-5094 on expression of STAT5b and its down-stream genes. (A) Expression of miR-5094 and mRNA levels of STAT5b, Bcl-2, Cyclin D1, Igf- 1 and p 21 in HeLa cells at $24 \mathrm{~h}$ after radiation. U6 and GADPH were used as controls. (B) Protein expression of STAT5b, Bcl-2, Cyclin DI, Iff-1 and p21 in HeLa cells at $24 \mathrm{~h}$ after radiation. NC: Negative mock control; 5094 mcs: miR-5094 mimics. $* \mathrm{P}<0.05$ and $* * \mathrm{P}<0.01$ represent the comparison with NC, while \#P $<0.05$ represent the comparison with NC plus 2 Gy $\mathrm{X}$-rays (NC-2Gy)

We also checked the growth of Jurkat T cells. As shown in Figure 4E, X-ray irradiation significantly suppressed cell proliferation of Jurkat cells. Transfection of miR-5094 mimics or STAT5b siRNA remarkably decreased Jurkat cells proliferation rate. However, miRNA inhibitor significantly improved cell proliferation, as compared with negative mock control, especially at $48 \mathrm{~h}$ post-irradiation.

We further validated STAT5b protein level in Jurkat $\mathrm{T}$ cells and HeLa cells. As shown in Figure 4F, in both HeLa and Jurkat T cell lines, miR-5094 mimics or STAT5b siRNA lowered expression of STAT5b with or without irradiation. Also, the inhibitor of miR-5094 resulted in upregulation of STAT5b.

\section{MiR-5094 suppresses human peripheral blood T cell proliferation}

Because of the key roles of STAT5b and Igf- 1 on lymphocyte proliferation and the maintenance of immune function, we further investigate the effect of miR-5094 in human peripheral blood T cells. CD4 ${ }^{+} \mathrm{T}$ cells and $\mathrm{CD} 4{ }^{+} \mathrm{CD} 25^{+}$Treg cells were separated from human peripheral blood and their growth curves were measured after irradiation and miRNAs transfection.

As shown in Figure 5A, 2 Gy of X-rays significantly suppressed proliferation of $\mathrm{CD} 4{ }^{+} \mathrm{CD} 25^{+}$Treg cells. As compared with mock control transfection, proliferation of $\mathrm{CD} 4{ }^{+} \mathrm{CD} 25^{+}$Treg cells was remarkably decreased at $72 \mathrm{~h}$ after miR-5094 transfection. Moreover, transfection of miR-5094 inhibitor mitigated the radiation-induced decline of Treg cells proliferation. Similarly, miR-5094 transfection and X-ray irradiation suppressed proliferation of $\mathrm{CD}^{+}{ }^{+} \mathrm{T}$ cells (Figure 5B). However, the transfection of miR-5094 inhibitor in $\mathrm{CD}^{+} \mathrm{T}$ cells did not rescue the compromised proliferation of $\mathrm{CD}^{+} \mathrm{T}$ cells after radiation. As expected, the proliferation of both $\mathrm{CD} 44^{+} \mathrm{CD} 25^{+}$Treg cells and $\mathrm{CD}^{+} \mathrm{T}$ cells decreased significantly without stimulation from anti-CD3/CD28 and IL-2 (Figure 5A and $5 \mathrm{~B})$.

The detection of $\mathrm{CD} 4{ }^{+} \mathrm{CD} 25^{+}$Treg proportion in all $\mathrm{CD}^{+} \mathrm{T}$ cells showed that $\mathrm{X}$-ray irradiation dramatically increased Treg proportions. More importantly, miR-5094 inhibitor further increased the percentage of $\mathrm{CD}_{4}^{+} \mathrm{CD} 25^{+}$Treg than radiation treatment alone (Figure 5C).

The expression of STAT5b and miR-5094 in CD4 ${ }^{+}$ $\mathrm{T}$ cells were next examined by qRT-PCR and Western blotting. The result showed that in $\mathrm{CD}^{+} \mathrm{T}$ cells, miR-5094 mimics or X-ray irradiation induced up-regulation of miR-5094 and down-regulation of STAT5b (Figure 5D). The miR-5094 inhibitor eliminated such an up-regulation or down-regulation (Figure 5D). Likewise, the Western blotting showed that the expression of STAT5b was suppressed by miR-5094 mimics or X-rays. Furthermore, the IR induced decrease in STAT5b was rescued by miR-5094 inhibitor. As expected, IL-2 stimulated the phosphorylation of STAT5b (Figure 5E).

\section{Discussion}

Our previous study demonstrated that miR-5094 is a novel human miRNA and detected its up-regulation after 2-Gy X-ray irradiation by qRT-PCR using stem-loop reverse transcription primers [16]. In this study, we confirmed responsive increase of miR-5094 in HeLa cells, Jurkat cells and human peripheral blood T cells after X-ray irradiation (Figure 2 and Figure 5). Additionally, we showed the expression levels of miR-5094 were elevated alongside with increasing X-ray dosage (Figure 2). These data verified miR-5094 is a radiation responsive miRNA and it is dramatically up-regulated by radiation treatment. Identifying target(s) of miR-5094, therefore, 
will advance current understanding of cellular radiation response.

Through TargetScan analysis, STAT5b was predicted as a putative target of miR-5094. Our reporter gene assay indeed showed that miR-5094 down-regulates reporter through the putative miR-5094 binding site in 3'-UTR of STAT5b. We further verified the suppression of STAT5b expression by miR-5094 mimics (Figure 1B and 1C). Moreover, our detection of miR-5094 and STAT5b expression at transcription and protein level after X-ray irradiation revealed an inversely correlation between STAT5b and miR-5094 (Figure 2A and 2B). These data suggested a regulatory link between miR-5094 and STAT5b.
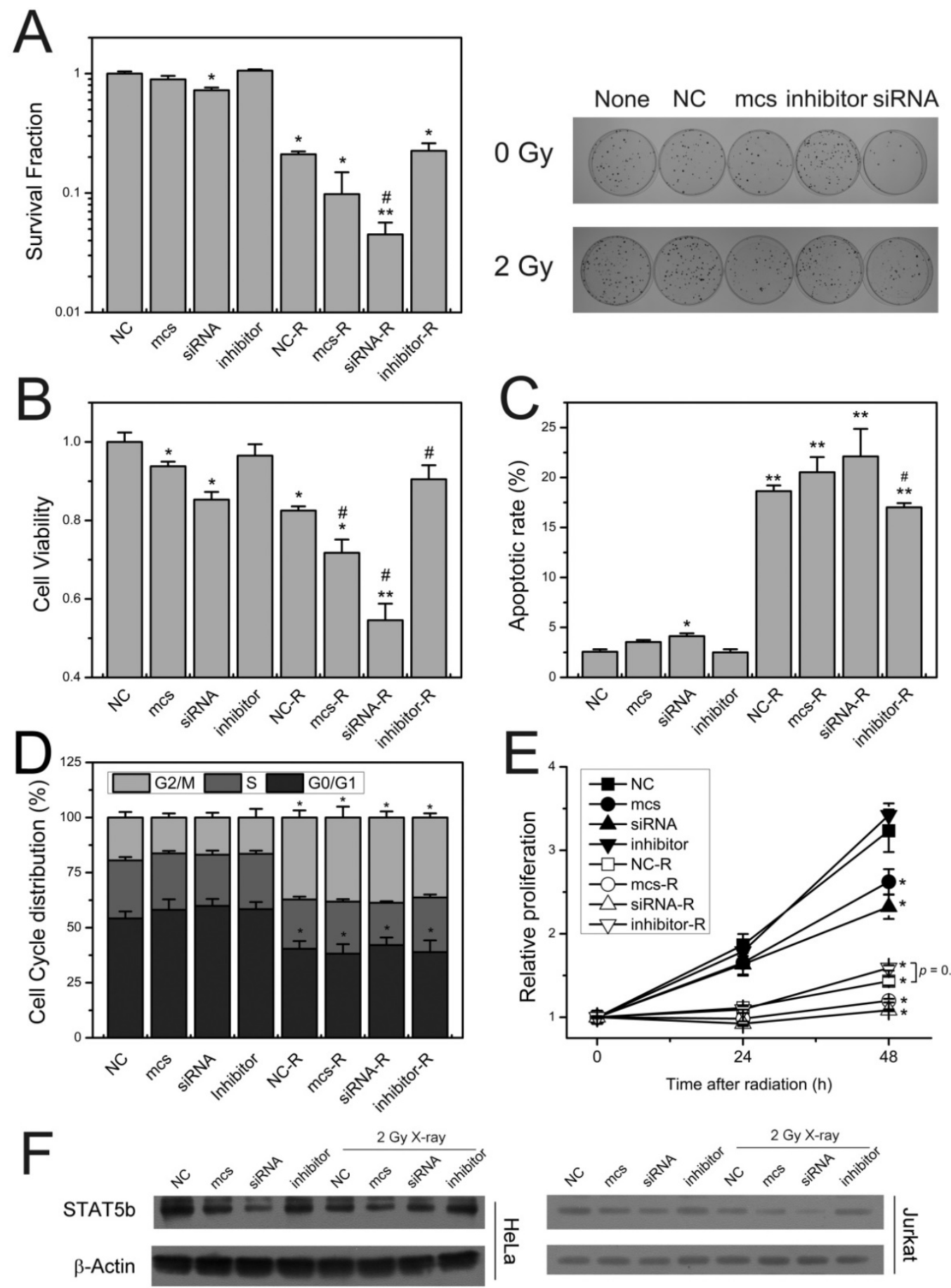

Figure 4. miR-5094 regulates cellular radiation response by targeting STAT5b. (A) Survival fraction of HeLa cells. Left graph shows quantitative bar of colony formation assay; right graph is plates of colony formation assay. (B) Cell viability assay of HeLa cells at $48 \mathrm{~h}$ after $2 \mathrm{~Gy} \mathrm{X}$-rays. (C) The apoptotic rate of HeLa cells at $24 \mathrm{~h}$ after 2 Gy X-rays. (D) Cell cycle distribution of HeLa cells treated with $2 \mathrm{~Gy}$ of X-rays at 24 h post-irradiation. (E) Proliferation curves of Jurkat cells. (F) The expression of STAT5b in HeLa cells and Jurkat cells at $24 \mathrm{~h}$ after radiation. None: non-transfected control; NC: negative mock control; mcs: miR-5094 mimics; siRNA: STAT5b siRNA; inhibitor: miR-5094 inhibitor. $* \mathrm{P}<0.05$ and $* * \mathrm{P}<0.01$ represent comparison with NC; \#P $<0.05$ represent comparison with NC plus 2 Gy $X-$ rays (NC-R). 


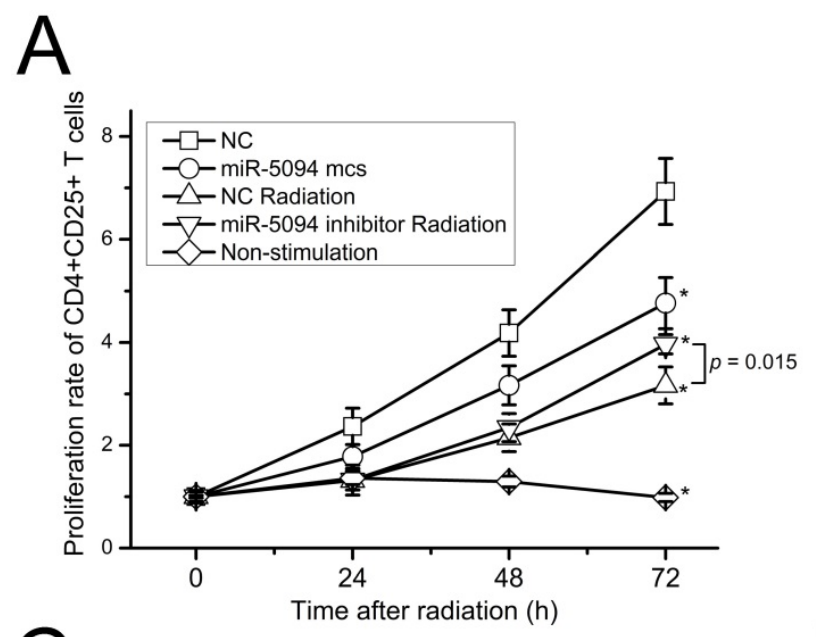

B
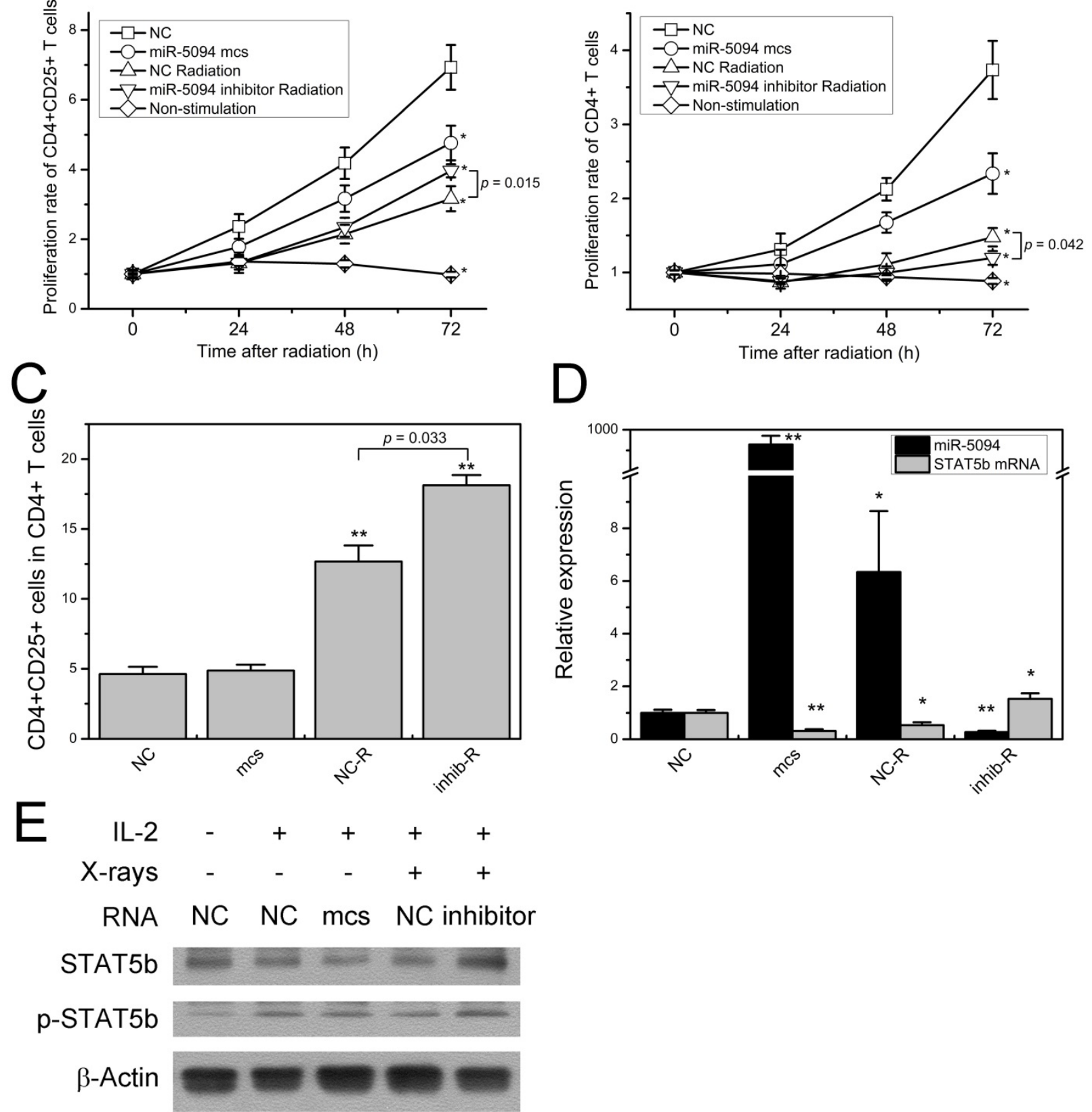

Figure 5. miR-5094 regulates $\mathbf{T}$ cells proliferation by targeting STAT5b. (A) Proliferation curves of CD4+CD25+ $T$ cells from human Peripheral blood. (B) Proliferation curves of CD4+ $T$ cells from human Peripheral blood. (C) Percentage of CD4 ${ }^{+} C D 25^{+} T$ cells in $C D 4+T$ cells at $72 \mathrm{~h}$ after radiation. (D) Expression of miR-5094 and STAT5b mRNA in CD4+ $\mathrm{T}$ cells at $24 \mathrm{~h}$ after radiation. $\mathrm{U} 6$ and GADPH mRNAs were used as controls. (E) Expression and activation of STAT5b in CD4+ $\mathrm{T}$ cells at $24 \mathrm{~h}$ after radiation. Cells were cultured with anti-CD3/CD28-coated beads (1:4) and subjected to IL-2 (20 U/mL) stimulation as indicated. NC: negative mock control; mcs: miR-5094 mimics; inhib: miR-5094 inhibitor; R: 2 Gy X-rays. ${ }^{\mathrm{P}}<0.05$ and $* * \mathrm{P}<0.01$ represent the comparison with NC.

It has been reported that STAT5b was also suppressed by other miRNAs, e.g., miR-134, miR-150-5p and miR-200a [30-32]. Moreover, it was reported that the expression of miR-134 was specifically up-regulated in human B lymphoblastic cell line IM9 at $24 \mathrm{~h}$ after $1 \mathrm{~Gy}$ and $10 \mathrm{~Gy} \gamma$-ray irradiation [34], and miR-200a showed a significant higher expression in patient saliva 12 months after radiotherapy [35]. These miRNAs were putative regulators of STAT5b after IR. Yet, no investigations have linked the expression changes of these miRNA to the changes of STAT5b in radiation response. Whereas, the data presents here showed no significant increase of miR-134, miR-150-5p and miR-200a in HeLa cells at $12 \mathrm{~h}$ and $24 \mathrm{~h}$ after X-ray irradiation (Figure 2C). According to recent studies on profiles of radiation responsive miRNA, the association between miRNA regulation and radiation exposure was showed to be dependent on the cell types, dosages and times after irradiation $[8,36,37]$. Likewise, Cha et 
al. reported 3.3-fold up-regulation of miR-134 and 2.6-fold down-regulation of miR-197 in IM9 cells at 24 $\mathrm{h}$ after 10 Gy $\gamma$-Ray irradiation [34], while they were not able to detect up-regulation of miR-134 but found 5.34-fold up-regulation of miR-197 in IM9 cells at $8 \mathrm{~h}$ after 10 Gy $\gamma$-Ray irradiation [38]. The inconsistency between previous research and our current study of miR-134, miR-150-5p and miR-200a may be due to the differences in cell types and time points after radiation used in studies.

Through regulation the expression of down-stream genes, STAT5b plays crucial role in cell growth, apoptosis and cell cycle arrest [21-23, 31, 39, 40]. Since these processes often take place during DDR, we hypothesized that miR-5094 may participates in cellular radiation response via STAT5b pathway. Our results showed that exogenous miR-5094 significantly suppressed cell proliferation of HeLa cells, Jurkat cells, CD4 ${ }^{+} \mathrm{CD} 25^{+}$Treg cells and $\mathrm{CD}^{+} \mathrm{T}$ cells (Figure 4B, 4D, 5A and 5B). Meanwhile, the proliferation suppressing effect induced by $\mathrm{X}$-ray irradiation were attenuated by miR-5094 inhibitor in HeLa cells, Jurkat cells, and $\mathrm{CD} 4{ }^{+} \mathrm{CD} 25^{+}$Treg cells (Figure $4 \mathrm{~B}$ and $5 \mathrm{~A}$ ). Furthermore, we validated the down-regulation of STAT5b by miR-5094 resulted in subsequent down-regulation of Bcl-2 and Igf-1 expression in both transcriptional and translational levels in HeLa cells after X-ray irradiation, and the transfection of miR-5094 inhibitor rescued the miR-5094-mediated expression suppression of STAT5b, Bcl-2 and Igf- 1 after irradiation (Figure 3). In the other hand, though the expression of Bcl-2, p21 and Cyclin D1 is changed in miR-5094 mimics transfection or miR-5094 inhibitor transfection groups (Figure 3B), we failed to detect remarkable increasing of G1 phase arrested cells nor apoptosis cells in miR-5094 mimics transfected groups (Figure 4C and $4 \mathrm{D})$, and the miR-5094 inhibitor did not show a significant influence on cell cycle distributions after X-ray irradiation. The probable reason for this result might be the effect of miR-5094 on cell cycle or apoptosis is so weak and is covered up by the intensive influence from ionizing radiation. Taken together, our data revealed that miR-5094 play a suppressive role in cell proliferation by targeting STAT5b during cellular radiation response.

Notably, the proliferation suppression of $\mathrm{CD}^{+} \mathrm{T}$ cells was not recovered by miR-5094 inhibitor after radiation, while the proliferation of $\mathrm{CD} 4{ }^{+} \mathrm{CD} 25^{+}$Treg cells was significantly increased under same treatment (Figure 5A and 5B). To understand this phenomenon, we subsequently detected the distribution of $\mathrm{CD}^{+} \mathrm{CD} 25^{+}$Treg cells in total $\mathrm{CD} 4^{+} \mathrm{T}$ cells. Results showed that radiation induced markedly increase of $\mathrm{CD} 4{ }^{+} \mathrm{CD} 25^{+}$Treg cells, while the miR-5094 inhibitor induced higher percentage of $\mathrm{CD} 4{ }^{+} \mathrm{CD} 25^{+}$ Treg cells after radiation (Figure 5C). These data coincident with previous studies that ionizing radiation impaired totally number of $\mathrm{CD} 4^{+} \mathrm{T}$ cells and $\mathrm{CD}^{+}{ }^{+} \mathrm{CD} 25^{+}$Treg cells $[41,42]$, while the ratio of Treg to $\mathrm{CD}^{+} \mathrm{T}$ cells is significantly enhanced as $\mathrm{CD} 4{ }^{+} \mathrm{CD} 25^{+}$Treg cells are more resistant to ionizing radiation than other $\mathrm{T}$ cells [41-43]. Additionally, though the immune suppression function of $\mathrm{CD} 4{ }^{+} \mathrm{CD} 25^{+}$Treg cells was impaired by radiation, the extent of the reduction was limited especially at lower dose according to different studies [41, 42, 44, 45]. Thus, the enhanced radiation sensitivity of total $\mathrm{CD} 4^{+}$ $\mathrm{T}$ cells in miR-5094 inhibition group may contributes to a higher percentage of $\mathrm{CD} 4{ }^{+} \mathrm{CD} 25^{+}$Treg cells after radiation (Figure 5C), which in turn suppressed the proliferation of $\mathrm{CD}^{+}{ }^{+} \mathrm{CD} 25^{-} \mathrm{T}$ cells. As the immunosuppressing effects of Treg cells always considered as side effect of radiation therapy [46], our data indicates miR-5094 may participates in the maintenance of immunosuppressing effects of Treg after IR. Altogether, our study uncovered the proliferation suppression function of radiation induced miR-5094 via STAT5b in $\mathrm{CD}^{+} \mathrm{CD} 25^{+}$Treg cells in vitro. Considering the difference between in vivo and in vitro system, further functional analysis will be necessary to elucidate the role of miR-5094 in Treg cells and immune system under ionizing radiation condition and we will work on it.

In summary, our study uncovered a role of radiation responsive miR-5094 in negative regulation of STAT5b protein level via miR-5094 binding site in STAT5b 3'-UTR. Our study also revealed that in cellular radiation response, negative regulation of STAT5b miR-5094 leads to subsequent inhibition of cell proliferation through the suppressing the transcription of STAT5b down-stream genes Bcl-2 and Igf-1. Our study provides a new insight into the role of miRNAs in STAT5b regulatory network in response to IR.

\section{Acknowledgements}

Jurkat cells were a kind gift from Dr. Yi Wu (School of life sciences, Lanzhou University, EBV-B cells from Dr. Zhikai Duan (China Institute for Radiation Protection, Taiyuan, China).

This work was supported by the National Key R\&D program of China (grant number: 2017YFC0 108602 and 2017YFC0108605), the National Nature Science Foundation of China (grant number: 11805246 and 31400723); the Hundred-Talent Program of Chinese Academy of Sciences (grant number: Y763050BRO); and the Science and Technology Research Project of Gansu Province (grant number: 145RTSA012). 


\section{Ethics Statement}

The experimental protocol was approved by the Medical Ethics Committee of Affiliation Hospital of Gansu University of Chinese Medicine, Lanzhou, China. The written informed consent was obtained from all five healthy volunteers.

\section{Competing Interests}

The authors have declared that no competing interest exists.

\section{References}

1. Shrivastav M, De Haro LP, Nickoloff JA. Regulation of DNA double-strand break repair pathway choice. Cell research. 2008; 18: 134-47.

2. Vatner RE, Cooper BT, Vanpouille-Box C, Demaria S, Formenti SC. Combinations of immunotherapy and radiation in cancer therapy. Front Oncol. 2014; 4: 325.

3. Lhuillier C, Rudqvist NP, Elemento O, Formenti SC, Demaria S. Radiation therapy and anti-tumor immunity: exposing immunogenic mutations to the immune system. Genome Med. 2019; 11: 40.

4. Ngwa W, Irabor OC, Schoenfeld JD, Hesser J, Demaria S, Formenti SC. Using immunotherapy to boost the abscopal effect. Nat Rev Cancer. 2018; 18: 313-22.

5. Grapin M, Richard C, Limagne E, Boidot R, Morgand V, Bertaut A, et al. Optimized fractionated radiotherapy with anti-PD-L1 and anti-TIGIT: a promising new combination. J Immunother Cancer. 2019; 7: 160.

6. Bartel DP. MicroRNAs: target recognition and regulatory functions. Cell. 2009; 136: 215-33

7. Zhao L, Lu X, Cao Y. MicroRNA and signal transduction pathways in tumor radiation response. Cellular signalling. 2013; 25: 1625-34.

8. $\mathrm{Hu}$ H, Gatti RA. MicroRNAs: new players in the DNA damage response. Journal of molecular cell biology. 2011; 3: 151-8.

9. Metheetrairut C, Slack FJ. MicroRNAs in the ionizing radiation response and in radiotherapy. Curr Opin Genet Dev. 2013; 23: 12-9.

10. Wagner-Ecker M, Schwager C, Wirkner U, Abdollahi A, Huber PE. MicroRNA expression after ionizing radiation in human endothelial cells. Radiation oncology. 2010; 5: 25.

11. Qu C, Liang Z, Huang J, Zhao R, Su C, Wang S, et al. MiR-205 determines the radioresistance of human nasopharyngeal carcinoma by directly targeting PTEN. Cell cycle. 2012; 11: 785-96.

12. Li B, Shi XB, Nori D, Chao CK, Chen AM, Valicenti $R$, et al. Down-regulation of microRNA 106b is involved in p21-mediated cell cycle arrest in response to radiation in prostate cancer cells. The Prostate. 2011; 71: 567-74.

13. Park M, Yoon HJ, Kang MC, Kwon J, Lee HW. MiR-338-5p enhances the radiosensitivity of esophageal squamous cell carcinoma by inducing apoptosis through targeting survivin. Scientific reports. 2017; 7: 10932.

14. He L, He X, Lim LP, de Stanchina E, Xuan Z, Liang Y, et al. A microRNA component of the p53 tumour suppressor network. Nature. 2007; 447: $1130-4$

15. Ji Q, Hao X, Zhang M, Tang W, Yang M, Li L, et al. MicroRNA miR-34 inhibits human pancreatic cancer tumor-initiating cells. PloS one. 2009; 4: e6816.

16. Ding N, Wu X, He J, Chang L, Hu W, Li W, et al. Detection of novel human MiRNAs responding to X-ray irradiation. Journal of radiation research. 2011; 52: 425-32.

17. Teglund S, McKay C, Schuetz E, van Deursen JM, Stravopodis D, Wang D, et al. Stat5a and Stat5b proteins have essential and nonessential, or redundant, roles in cytokine responses. Cell. 1998; 93: 841-50.

18. Hwa V. STAT5B deficiency: Impacts on human growth and immunity. Growth hormone \& IGF research : official journal of the Growth Hormone Research Society and the International IGF Research Society. 2016; 28: 16-20.

19. Kanai T, Jenks J, Nadeau KC. The STAT5b Pathway Defect and Autoimmunity. Front Immunol. 2012; 3: 234

20. Varco-Merth B, Rotwein P. Differential effects of STAT proteins on growth hormone-mediated IGF-I gene expression. Am J Physiol Endocrinol Metab. 2014; 307: E847-55.

21. Liang QC, Xiong H, Zhao ZW, Jia D, Li WX, Qin HZ, et al. Inhibition of transcription factor STAT5b suppresses proliferation, induces G1 cell cycle arrest and reduces tumor cell invasion in human glioblastoma multiforme cells. Cancer Lett. 2009; 273: 164-71.
22. Joung YH, Lee MY, Lim EJ, Kim MS, Hwang TS, Kim SY, et al. Hypoxia activates the IGF-1 expression through STAT5b in human HepG2 cells. Biochem Biophys Res Commun. 2007; 358: 733-8.

23. O'Brien K, Lowry MC, Corcoran C, Martinez VG, Daly M, Rani S, et al. miR-134 in extracellular vesicles reduces triple-negative breast cancer aggression and increases drug sensitivity. Oncotarget. 2015; 6: 32774-89.

24. Jin Y, Purohit S, Chen X, Yi B, She JX. Over-expression of Stat $5 b$ confers protection against diabetes in the non-obese diabetic (NOD) mice via up-regulation of $\mathrm{CD} 4(+) \mathrm{CD} 25(+)$ regulatory $\mathrm{T}$ cells. Biochem Biophys Res Commun. 2012; 424: 669-74.

25. Cohen AC, Nadeau KC, Tu W, Hwa V, Dionis K, Bezrodnik L, et al. Cutting edge: Decreased accumulation and regulatory function of CD4+ CD25(high) $\mathrm{T}$ cells in human STAT5b deficiency. Journal of immunology. 2006; 177: 2770-4.

26. Kulms D, Schwarz T. Ultraviolet radiation inhibits interleukin-2-induced tyrosine phosphorylation and the activation of STAT5 in T lymphocytes. The Journal of biological chemistry. 2001; 276: 12849-55.

27. Hu ZZ, Huang $\mathrm{H}$, Cheema A, Jung M, Dritschilo A, Wu CH. Integrated Bioinformatics for Radiation-Induced Pathway Analysis from Proteomics and Microarray Data. Journal of proteomics \& bioinformatics. 2008; 1: 47-60

28. Edmondson DA, Karski EE, Kohlgruber A, Koneru H, Matthay KK, Allen S, et al. Transcript Analysis for Internal Biodosimetry Using Peripheral Blood from Neuroblastoma Patients Treated with (131)I-mIBG, a Targeted Radionuclide. Radiation research. 2016; 186: $235-44$.

29. Herok R, Konopacka M, Polanska J, Swierniak A, Rogolinski J, Jaksik R, et al. Bystander effects induced by medium from irradiated cells: similar transcriptome responses in irradiated and bystander K562 cells. International journal of radiation oncology, biology, physics. 2010; 77: 244-52.

30. Williams KC, Renthal NE, Condon JC, Gerard RD, Mendelson CR. MicroRNA-200a serves a key role in the decline of progesterone receptor function leading to term and preterm labor. Proceedings of the National Academy of Sciences of the United States of America. 2012; 109: 7529-34.

31. Zhang Y, Kim J, Mueller AC, Dey B, Yang Y, Lee DH, et al. Multiple receptor tyrosine kinases converge on microRNA-134 to control KRAS, STAT5B, and glioblastoma. Cell death and differentiation. 2014; 21: $720-34$

32. Heinz RE, Rudolph MC, Ramanathan P, Spoelstra NS, Butterfield KT, Webb PG, et al. Constitutive expression of microRNA-150 in mammary epithelium suppresses secretory activation and impairs de novo lipogenesis. Development. 2016; 143: 4236-48.

33. Peng H, Liu Y, Tian J, Ma J, Tang X, Rui K, et al. The Long Noncoding RNA IFNG-AS1 Promotes T Helper Type 1 Cells Response in Patients with Hashimoto's Thyroiditis. Scientific reports. 2015; 5: 17702.

34. Cha HJ, Shin S, Yoo H, Lee EM, Bae S, Yang KH, et al. Identification of ionizing radiation-responsive microRNAs in the IM9 human B lymphoblastic cell line. International journal of oncology. 2009; 34: $1661-8$.

35. Greither T, Vorwerk F, Kappler M, Bache M, Taubert H, Kuhnt T, et al. Salivary miR-93 and miR-200a as post-radiotherapy biomarkers in head and neck squamous cell carcinoma. Oncology reports. 2017; 38: 1268-75.

36. Maes OC, An J, Sarojini H, Wu H, Wang E. Changes in MicroRNA expression patterns in human fibroblasts after low-LET radiation. J Cell Biochem. 2008; 105: 824-34

37. Wei W, He J, Wang J, Ding N, Wang B, Lin S, et al. Serum microRNAs as Early Indicators for Estimation of Exposure Degree in Response to Ionizing Irradiation. Radiation research. 2017; 188: 342-54

38. Cha HJ, Seong KM, Bae S, Jung JH, Kim CS, Yang KH, et al. Identification of specific microRNAs responding to low and high dose gamma-irradiation in the human lymphoblast line IM9. Oncology reports. 2009; 22: 863-8.

39. Hwa V, Nadeau K, Wit JM, Rosenfeld RG, STAT5b deficiency: lessons from STAT5b gene mutations. Best practice \& research Clinical endocrinology \& metabolism. 2011; 25: 61-75.

40. Nadeau K, Hwa V, Rosenfeld RG. STAT5b deficiency: an unsuspected cause of growth failure, immunodeficiency, and severe pulmonary disease. The Journal of pediatrics. 2011; 158: 701-8.

41. Qu Y, Jin S, Zhang A, Zhang B, Shi X, Wang J, et al. Gamma-ray resistance of regulatory CD4+CD25+Foxp3+ T cells in mice. Radiation research. 2010; 173: 148-57.

42. Qu Y, Zhang B, Liu S, Zhang A, Wu T, Zhao Y. 2-Gy whole-body irradiation significantly alters the balance of CD4+ CD25- T effector cells and CD4+ CD25+ Foxp3+ T regulatory cells in mice. Cell Mol Immunol. 2010; 7: 419-27.

43. Kachikwu EL, Iwamoto KS, Liao YP, DeMarco JJ, Agazaryan N, Economou JS, et al. Radiation enhances regulatory $\mathrm{T}$ cell representation. 
International journal of radiation oncology, biology, physics. 2011; 81: 1128-35.

44. Cao M, Cabrera R, Xu Y, Liu C, Nelson D. Gamma irradiation alters the phenotype and function of CD4+CD25+ regulatory T cells. Cell Biol Int. 2009; 33: 565-71.

45. Balogh A, Persa E, Bogdandi EN, Benedek A, Hegyesi H, Safrany G, et al. The effect of ionizing radiation on the homeostasis and functional integrity of murine splenic regulatory T cells. Inflamm Res. 2013; 62: 201-12.

46. Muroyama Y, Nirschl TR, Kochel CM, Lopez-Bujanda Z, Theodros D, Mao W, et al. Stereotactic Radiotherapy Increases Functionally Suppressive Regulatory T Cells in the Tumor Microenvironment. Cancer Immunol Res. 2017; 5: 992-1004. 